\title{
AMEC multigas passive sampler: a green product for cost-effectively monitoring air pollution indoors and outdoors
}

\author{
H. Tang, L. Burns, L. Yang \& F. Apon \\ AMEC Earth and Environmental, Alberta, Canada
}

\begin{abstract}
Climate change, sustainable development, and greenhouse gas are several of the hot topics in the world. Saving our limited resources, reducing consumption, and waste are emergent tasks facing the world. As a result, a new generation of passive sampling technology - multi-gas passive sampling system (MGPS) has been developed and reported here. This paper will demonstrate the cost effective unique features of the MGPS compared with many normal passive samplers (NPS). Cross contamination problems have been comprehensively studied and reported in this paper.
\end{abstract}

Keywords: air pollution, passive sampler, air monitoring.

\section{Introduction}

Air pollution indoors and outdoors has become a health issue in the world. Scientific and social interest in monitoring air pollutants indoors and outdoors is increasing. Thus, saving our limited resources, reducing consumption, and reducing waste are emergent tasks facing the world environmental business. Many monitoring technologies for air pollutants have been developed and subsequently improved in the past few decades. Due to its cost effective and more convenient to use, passive sampling technology is becoming more and more popular. In the past decades, many different types of passive samplers have been developed.

The first passive sampler in the world was used by Fox [1] in 1873 for monitoring ozone concentrations. Since then, passive samplers have been developed for monitoring air pollution in the ambient environment (including vegetation canopies study), work place, and indoor environment, which include 
air pollutants such as $\mathrm{SO}_{2}[2,3], \mathrm{NO}_{2}[4,5], \mathrm{H}_{2} \mathrm{~S}[6,7], \mathrm{NO}_{\mathrm{x}}$ [8], $\mathrm{O}_{3}[9]$, VOC [10], aldehyde [11] etc.

The sampling rate is a key parameter related to the correct measurement of air pollutants using passive samplers. Active samplers have a known sampling rate, which is the pump's flow rate. The passive sampler's sampling rates depend on many factors such as temperature, relative humidity, wind direction, wind speed, sampler's structure, collection media etc. If a passive sampler can be used in all climate conditions, a fixed passive sampling rate obtained from laboratories cannot be used for ambient studies anywhere and anytime in the world. It would be highly unreasonable to expect that a passive sampler's sampling rate would be the same when temperatures change from $-30^{\circ} \mathrm{C}$ to $+30^{\circ} \mathrm{C}$ and relative humidity change from $90 \%$ to $15 \%$. Therefore, the key factor for using passive samplers is how to determine their sampling rates. Tang et al. have reported using equations to address the problems [12-15] Field applications proved that Tang's approach was practically useful in many cases.

Krupa and Legge [16] have summarized the passive samplers into different types, such as badge, diffusion tube with filter absorption or solid absorption, and adsorption cartridge etc. The all season passive sampling system (ASPS) designed by Tang et al. [12, 13, 15] and Tang and Lau [14] (Figure 1) is a mixture of bandage and cartridge which can be reused many times; a rain shelter is designed to hold three passive samplers for triplicate study.

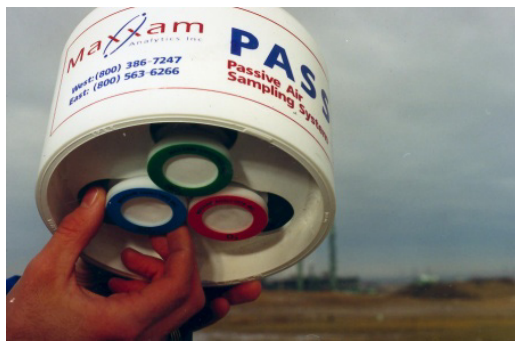

Figure 1: The all-season passive air sampling system designed by Tang.

So far, in all the badge and cartridge type of passive samplers described above, each one can only be used to collect one air pollutant (such as $\mathrm{SO}_{2}, \mathrm{NO}_{2}$, $\mathrm{NO}_{\mathrm{x}}, \mathrm{H}_{2} \mathrm{~S}, \mathrm{O}_{3}, \mathrm{NH}_{3}$ etc) or a group of air pollutants (such as volatile organic compounds (VOC) or aldehyde and ketone). In practice, field studies are requested to monitor several air pollutants in replicated in order to increase confidence level. For example, if 4 air pollutants are monitored at the same time and the same location in triplicate, 3 rain-shelters and 12 passive samplers plus several blanks will be used. This operation is tedious. In order to reduce cost and save our environment, several scientists have studied different ways to address the above problems. Tang et al. have tested a collection medium which can be used to simultaneously sample $\mathrm{HF}, \mathrm{NO}_{2}$ and $\mathrm{SO}_{2}$ (Tang 2010). In the Ogawa passive sampler, due to two separate sampling chambers in each side, thus, it can 
be easily installed two different collection media. A study for sampling $\mathrm{NO}_{2}$ and $\mathrm{NO}_{\mathrm{x}}$ together has been reported [17].

A new generation of passive sampling technology - multigas passive sampling system (MGPS) has been developed and validated. In the MGPS, one to several collection media can be packed into one passive sample body. Compared to the normal passive samplers (NPS), the MGPS is environment friendly, more cost-effective, more convenient to use, more accurate, and more flexible. This paper will report the features of MGPS and the field study results.

\section{The multigas passive sampling system}

\subsection{Principle of passive sampler}

It is well known that a passive (or diffusive) sampler is a device which is capable of taking samples of gas or vapor pollutants from air at a rate controlled by a physical process such as diffusion through a static air layer or permeation through a membrane.

The collected amount of an air pollutant by a passive sampler can be derived from Equation (1).

$$
Q=C D A^{t}
$$

where $\mathrm{Q}$ is the amount of the air pollutant collected by the passive sampler, $\mathrm{t}$ is the sampling time.

From Equation (2), it can be seen that the collection amount is proportional to the collection medium area. Theoretically, if only one quarter of the collection medium is used in the same passive sampler, the passive sampler should only collect one quarter amount of the air pollutant collected by the whole filter. Equation (2) is the principle of the multigas passive sampler.

\subsection{Multigas passive sampling system}

Different from the NPS that use one collection medium for collecting a single or a group of air pollutants such as active charcoal for volatile organic compounds (VOC), the multigas passive sampling system (MGPS) (Figure 2) uses one passive sampler body to pack several different collection media at the same time to collect several air pollutants. In the MGPS, VOC or aldehyde is considered as one (group) air pollutant.

The AMEC MGPS passive sampler body with a newly designed insert is shown in Figure 3. The MGPS can be used for both indoor, ambient air quality and personal exposure studies. The insert can be used to install 4 different collection media as the first layer in the passive sampler body. For more pollutants, a second layer or more layers can also be used. In this paper, we only report the first layer study. 


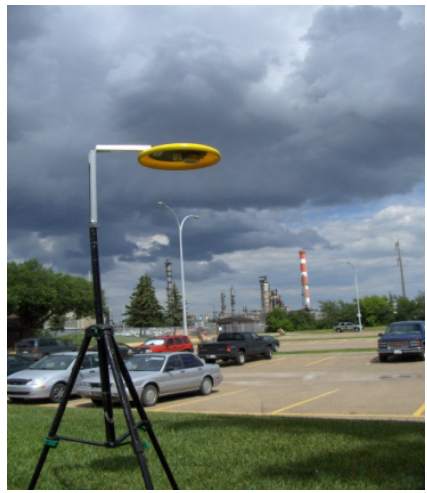

Figure 2: $\quad$ AMEC multigas passive sampling system.

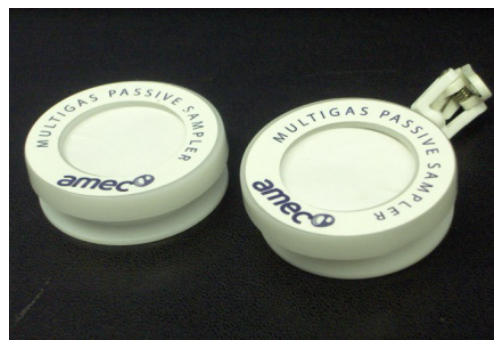

Figure 3: AMEC passive samplers for indoor, ambient and personal exposure uses.

The advantages of the MGPS include:

Environment friendly, which can save materials, chemicals, wastes, etc., cost effective, convenient to use, which can save labor for field jobs and passive sampler management,

flexible for clients, in which one to several collection filters can be installed based on clients' need, more accurate.

Table 1 lists comparisons of sampling 4 air pollutants in triplicate by using MGPS and NPS.

\subsection{Collection media preparation and sample analyses}

The following air pollutants were studied in this report: $\mathrm{SO}_{2}, \mathrm{NO}_{2}, \mathrm{O}_{3}$, and $\mathrm{H}_{2} \mathrm{~S}$. The collection media preparations were following References 15, 20, 17, and 23 respectively. All the chemicals used in this study were purified grade (Fisher Scientific, Nepean, CA). 
Table 1: $\quad$ Four air pollutants collected in triplicate by NPS and MGPS.

\begin{tabular}{|c|c|c|c|c|}
\hline ITEM & No. for NPS & No. for MGPS & No. Saved & \% Saved \\
\hline Rain shelter & 4 & 1 & 3 & 75 \\
\hline Passive body & 12 & 3 & 9 & 75 \\
\hline Diffusion Barrier & 12 & 3 & 9 & 75 \\
\hline Collection filter & 12 & 3 & 9 & 75 \\
\hline Chemicals* & 12 & 3 & 9 & 75 \\
\hline Waste generated** & 12 & 3 & 9 & 75 \\
\hline Field installation & 12 & 3 & 9 & 75 \\
\hline Average & & & & 75 \\
\hline
\end{tabular}

*Comparison of chemicals implies total chemicals used for one collection medium as one unit. In this case, 12 units are used for NPS; only 3 are used for MGPS.

**Comparison of waste generated uses extraction volume for one collection medium as one unit. In this case, 12 collection media are used by NPS, which generate 12 unit wastes; but only 3units are generated in MGPS.

Analyses of extractions of $\mathrm{SO}_{2}, \mathrm{NO}_{2}$, and $\mathrm{O}_{3}$ collection media were used IC. The $\mathrm{H}_{2} \mathrm{~S}$ collection medium extraction was analyzed by a filter fluorometer.

\subsection{Field validation}

The MGPS passive samplers were installed in six locations in Alberta (Figure 4). Duplicate or triplate passive samplers and duplicate field blanks were used. One location was at the Alberta Environmental (ANEV) industrial monitoring site in Edmonton (EIMU) which is equipped with a $\mathrm{NO}_{\mathrm{x}}$ continuous analyzer (TECO Model 42, Thermo Environmental Instruments Inc., Franklin MA), an $\mathrm{SO}_{2}$ analyzer (TECO 45C), an $\mathrm{O}_{3}$ analyzer (TECO 49), a $\mathrm{H}_{2} \mathrm{~S}$ analyzer (TECO Model 45C), a temperature measurement device (Model 41372 Campbell Scientific Inc., Logan UT), a relative humidity measurement device (Model 41372 Campbell Scientific Inc., Logan UT), and a wind speed monitoring device (Wind Flo 540, Athabasca Research Ltd., Edmonton, AB). The other five locations were at the Parkland Airshed Management Zone (PAMZ) monitoring site near Red Deer (RD), West Central Airshed Society (WACS) monitoring stations near Breton (BT) and Carrot Creek (CC), and ANEV industrial monitoring site in Calgary (CIMU) and Lethbridge. Those stations were equipped with similar devices as in the EIMU. Except in BT and CC, the rain shelters were fastened using an outside bracket so that the passive samplers were at almost the same elevation as the inlet for the air pollutant continuous analyzers (Figure 5). In BT and $\mathrm{CC}$, the rainshelters were installed in the fences about 2 meters above the ground. 


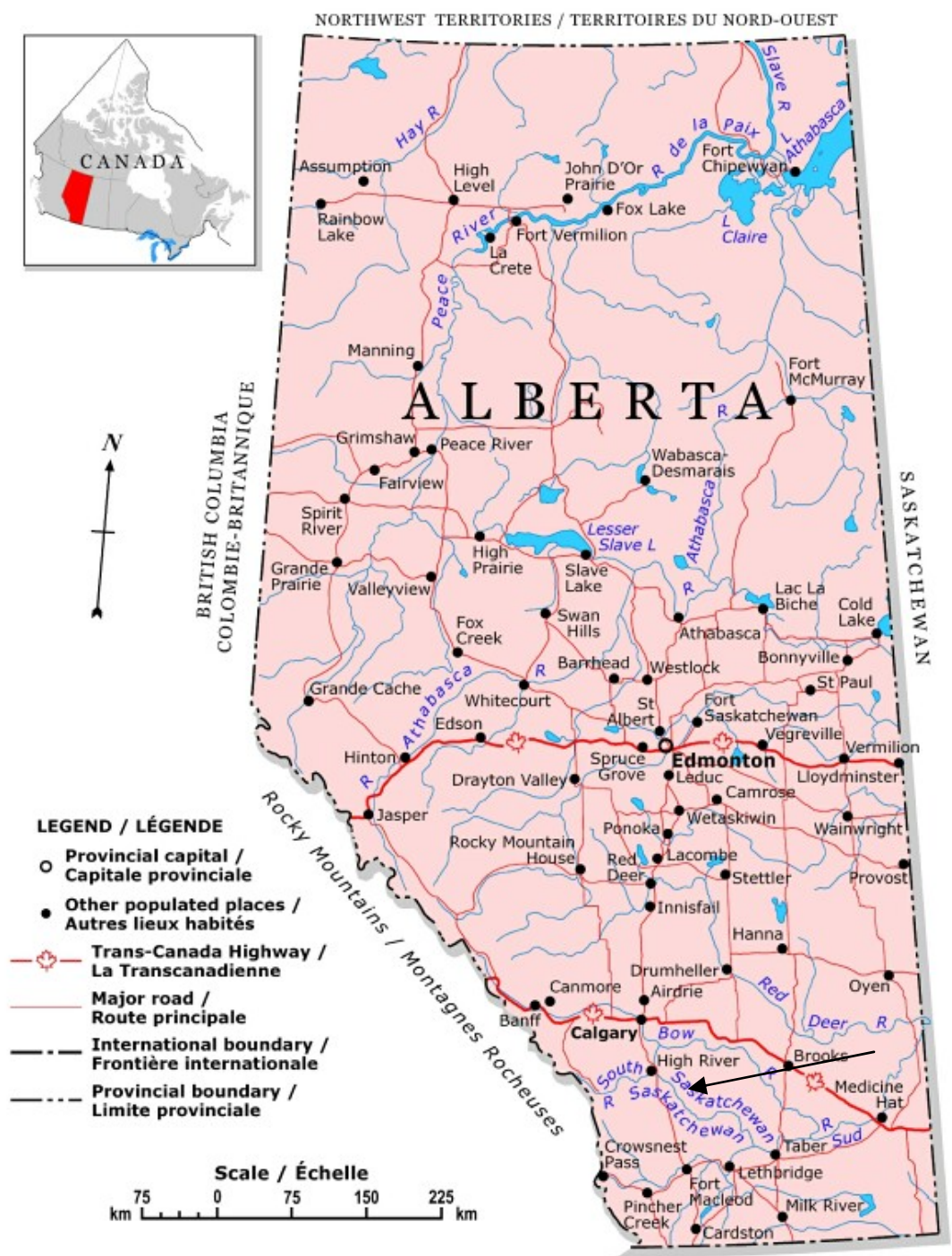

(c) 2001. Her Majesty the Queen in Right of Canada, Natural Resources Canada.

U S A / É-U d'A Sa Majesté la Reine du chef du Canada, Ressources naturelles Canada.

Figure 4: Alberta locations for installing MGPS.

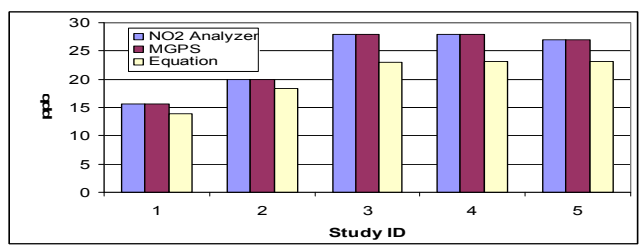

Figure 5: Comparison of $\mathrm{O}_{3}$ concentrations in EIMU. 


\section{Results and discussion}

\subsection{Different collection filter size studies and sampling rate determination}

In order to evaluate Equation (2), filters with $37 \mathrm{~mm}$ diameter and quarter size of the $37 \mathrm{~mm}$ filters prepared for sampling $\mathrm{SO}_{2}$ and $\mathrm{H}_{2} \mathrm{~S}$ were packed in the MGPS and installed in EIMU. Results are shown in Table 2.

Table 2: $\quad$ Collection quantities of different size filters in the MGPS.

\begin{tabular}{|c|c|c|}
\hline Filter size & $\mathrm{SO}_{2} \mu \mathrm{g}$ & $\mathrm{H}_{2} \mathrm{~S} \mathrm{ng}$ \\
\hline 37 mm filter (A) & 1.8 & 129.9 \\
\hline Quarter 1 of A & 0.5 & 37.2 \\
\hline Quarter 2 of A & 0.5 & 36.3 \\
\hline Quarter 3 of A & 0.5 & 34.5 \\
\hline Quarter 4 of A & 0.6 & 35.1 \\
\hline Sum of 4 quarter filter & 2.1 & 142.9 \\
\hline
\end{tabular}

The data in Table 2 demonstrate that reducing size of collection filter in the MGPS is applicable. The collection efficiencies inside the MGPS among the four quarter filters are almost the same since the relative standard deviations are only 3 and $4 \%$ for $\mathrm{SO}_{2}$ and $\mathrm{H}_{2} \mathrm{~S}$ respectively. The collection quantities of $37 \mathrm{~mm}$ filters for both $\mathrm{SO}_{2}$ and $\mathrm{H}_{2} \mathrm{~S}$ are a little bit lower compared to the sum of 4 quarter filters. Besides analytical method deviations, the other reason might be the increase of the quarter filters' surface area after the $37 \mathrm{~mm}$ filters were cut to quarter size.

The above results also indicate that the MGPS sampling rate can be calculated by the equations published by Tang before. For example, the ozone sampling rate for ASPS is shown in the following equation [14].

$$
\mathrm{R}_{\mathrm{S}}=14.8 \mathrm{~T}^{1 / 2}+0.259 \mathrm{RH}+0.275 \mathrm{WSP}-197
$$

where $\mathrm{R}_{\mathrm{S}}$ is the ozone sampling rate, $\mathrm{ml} / \mathrm{min}$; $\mathrm{T}$ is average temperature of the sampling period, K; RH is average humidity (\%); WSP is average wind speed, $\mathrm{cm} / \mathrm{sec}$, if WSP $>130$, then WSP $=130$. A quarter of the ASPS sampling rate theoretically can be the pollutant sampling rate in the MGPS. The sampling rate calculation equations for $\mathrm{NO}_{2}$ and $\mathrm{H}_{2} \mathrm{~S}$ have been changed since the collection media for those pollutants were different compared to references 13 and 15.

Actually the MGPS sampling rate determinations now are using a new approach which is named as "Integrative Passive Network Data Management" (IPN). The new approach makes the passive results more reasonable and accurate.

\subsection{Practical quantitative detection limit}

Tang et al. have reported practical quantitative determination limit (PQDL) for the all season passive sampling system before. Those PQDL were based on 
laboratory filter blank studies. For example, it was found that the pooled standard deviation was $0.6 \mu \mathrm{g}$ of nitrate per ozone collection filter based on a 24 hour exposure. The practical quantitative detection limit, thus, can be taken as $6 \mu \mathrm{g}$ per filter (10 times the standard deviation). This is equivalent to exposure of the passive sampler to $3 \mathrm{ppb} \mathrm{O}_{3}$ in the atmosphere for 24 hours. If the exposure period were increased to one month (30 days), the method practical quantitative detection limit for $\mathrm{O}_{3}$ in the atmosphere would be about $0.1 \mathrm{ppb}$. The collection filter's area in the MGPS is quarter of the filter in the ASPS. It is easy to obtain the same PQDL through reducing the extract volume to be used in the MGPS filter. For example, the extraction volume for ozone collection filter in the ASPS is $20 \mathrm{ml}$ of DI water. If the volume is reduced to $5 \mathrm{ml}$ in the MGPS, the PQDL for the MGPS will be the same as for the ASPS.

\subsection{Interference}

Numerous reagents have been checked for possible interference in the MGPS. It is found that if carefully choosing collection media and separating the media using different methods, there will be no substantial interferences.

A number of studies were conducted in the AMEC laboratory to study the interference among the different collection media. The studies were conducted by using one MGPS passive sampler packed all collection media and only one collection medium (duplicate) packed in one passive sampler.

Studies found that several chemicals could cause interferences to other pollutants' detection. For example, based on properties of chemicals used to collect air pollutants, nitric acid in the $\mathrm{H}_{2} \mathrm{~S}$ paper is expected to cause interference problems since it can generate nitric acid vapor, which can react metal parts in the passive sampler and can be adsorbed by filter papers. In the ozone passive samplers, ozone is reacted with nitrite in the collection paper, and the react product is nitrate. The nitrate concentration in the ozone passive sampler is used to calculate ozone concentration in air. Therefore, nitric acid can directly cause positive interference for ozone collection. Laboratory filter blank studies reflected the theoretical analysis above. Table 3 lists nitric concentrations measured in different ozone collection filter blanks.

Table 3: $\quad$ Nitric concentrations of different ozone collection filters.

\begin{tabular}{cccccc}
\hline $\begin{array}{c}\text { Test } \\
\text { No. }\end{array}$ & $\begin{array}{c}\text { Blank } \\
\text { filters* }\end{array}$ & $\begin{array}{c}\text { Stored } \\
\text { time } \\
\text { (Day) }\end{array}$ & \multicolumn{2}{c}{$\begin{array}{c}\text { Nitrate concentration } \\
\text { (ug/filter) }\end{array}$} & $\begin{array}{c}\text { Error } \\
\%\end{array}$ \\
\cline { 4 - 5 } & & & $\begin{array}{c}\text { Room } \\
\left(21^{\circ} \mathrm{C}\right)\end{array}$ & $\begin{array}{c}\text { Refrigerator } \\
\left(-4^{\circ} \mathrm{C}\right)\end{array}$ & \\
\hline 1 & $A$ & 14 & 0.56 & 0.54 & 4 \\
2 & $B$ & 14 & 1.30 & 0.55 & 136 \\
3 & $C$ & 21 & 0.65 & 0.61 & 6 \\
4 & $D$ & 21 & 0.96 & 0.56 & 71 \\
5 & $E$ & 21 & 1.26 & 0.55 & 129 \\
\hline
\end{tabular}

*Blank filters were from different MGPS passive samplers. For detail, refer to the following paragraph. 
In Table 1, $A$ blank filters were from MGPS passive samplers packed into only two ozone collection filters. Therefore there were no significant differences of nitric concentrations found between passives stored at room temperature and refrigerator. $B$ blank filters were from MGPS passive samplers packed into two ozone collection filters and two $\mathrm{H}_{2} \mathrm{~S}$ collection filters (coated with silver nitrate with $0.01 \mathrm{~N}$ nitric acid) in one sampler. It is clear that nitric acid vapor had adsorbed in the ozone collection filters; but at cooled temperature, the interference was not substantial. $C, D, E$ blank filters were from ozone collection filters packed into MGPS passive samplers together with $\mathrm{H}_{2} \mathrm{~S}$ filters coated with solutions with $0.001 \mathrm{~N}, 0.004 \mathrm{~N}$, and $0.01 \mathrm{~N}$ nitric acid respectively. Although all ozone filters stored at refrigerator had not been affected by $\mathrm{H}_{2} \mathrm{~S}$ filters, nitrate concentrations in the ozone filters stored at room temperature did indeed decrease along with the decrease of nitric acid concentrations in the coating solutions.

A new $\mathrm{H}_{2} \mathrm{~S}$ passive sampler without nitric acid is being continuously developed in the AMEC Centre for Passive Sapling Technology.

\subsection{Field study results}

\subsubsection{EIMU studies}

Many MGPS studies have been conducted in EIMU. For example, several studies results for $\mathrm{O}_{3}$ are listed in Table 4. The study periods, meteorological conditions, calculated sampling rates, MGPS sampling rates, and relative errors in each study are also listed in the tables.

Reid [18] conducted a study in 2000 using ASPS for monitoring $\mathrm{SO}_{2}$ concentrations in the Northern Rocky Mountain foothill of BC Canada. He found the $\mathrm{SO}_{2}$ concentrations were substantially different between continuous analyzer results and the ASPS results. But both the $\mathrm{SO}_{2}$ concentration trends kept the same. In this study, we met the same problems. Figure 5 shows comparisons of $\mathrm{O}_{3}$ concentrations obtained by analyzers, calculated from equations, and from the MGPS. It can be found that there are good agreements between results from

Table 4: $\quad$ Study results in EIMU for $\mathrm{O}_{3}$ by MGPS.

\begin{tabular}{|c|c|c|c|c|c|c|c|c|c|}
\hline \# & Location & Day & Date & $\begin{array}{l}\text { RH } \\
\%\end{array}$ & $\begin{array}{c}\mathrm{T} \\
{ }^{\circ} \mathrm{C}\end{array}$ & $\begin{array}{l}\text { WSP } \\
\mathrm{Km} / \mathrm{h}\end{array}$ & $\begin{array}{l}\text { Cal. } R_{S^{*}} \\
\mathrm{~cm} / \mathrm{min}\end{array}$ & $\begin{array}{l}\text { MGPS } \\
\text { Rs } \\
\mathrm{cm} / \mathrm{min}\end{array}$ & $\begin{array}{c}\text { Error } \\
\%\end{array}$ \\
\hline 1 & EIMU $^{*}$ & 6 & Oct 3-9 & 61 & 8 & 10 & 24.1 & 21.5 & 10.8 \\
\hline 2 & EIMU & 3 & Oct $26-29$ & 55 & 7 & 11 & 23.8 & 19 & 20.2 \\
\hline 3 & EIMU & 14 & Dec 3-17 & 79 & -9 & 8 & 22.9 & 24 & -4.8 \\
\hline 4 & EIMU & 17 & $\begin{array}{c}\text { Dec 17- } \\
\text { Jan } 3\end{array}$ & 82 & -9 & 7 & 23.1 & 21 & 9.1 \\
\hline 5 & EIMU & 21 & Jan 3-23 & 79 & -8 & 7 & 23.1 & 22 & 4.8 \\
\hline
\end{tabular}


analyzers and MGPS, but difference between analyzers and equations although the concentration trends are the same, in which when temperature decreased the concentrations of $\mathrm{NO}_{2}$ increased and the concentrations of $\mathrm{O}_{3}$ decreased. A further discussion for the sampling rate will be continued in the next section.

\subsubsection{Comparison of EIMU, CIMU, BT, CC, LG and RD studies}

In February 2008, the MGPS were installed in EIMU, CIMU, CC, BT, LG and RD. Table 5 summarized the weather conditions, calculated sampling rates using equations, MGPS sampling rates and the relative errors in each parameter.

Table 5: $\quad$ Comparison of NPS and MGPS.

\begin{tabular}{|l|l|l|l|}
\hline ITEM & NPS & MGPS & SAVE \% \\
\hline Pass body & 95 & 34 & 64 \\
\hline Diffusion barrier & 95 & 34 & 64 \\
\hline Collection filter & 95 & 24 & 75 \\
\hline Field job & 95 & 34 & 64 \\
\hline Waste generated & 95 & 24 & 75 \\
\hline
\end{tabular}

It can be seen that the weather conditions from EIMU to CIMU in the study period had no substantial difference. Therefore, the calculated sampling rates from pollutant to pollutant did not vary a lot. But the concentrations obtained by using equations had relatively large difference compared to the results obtained from analyzers and the MGPS (Figure 6).

It is well known that passive sampling rates depend on many factors. In addition to temperature, relative humidity, and wind speed, many other factors such as atmospheric pressure, local terrain, chemicals in the atmosphere etc. also play important roles. Tang et al. simplified the sampling rate calculation through only using temperature, relative humidity, and wind speed, in many cases, it did provide a useful tool for accurately monitoring air quality; in some other cases it might generate large deviation, which has been discussed before. We are conducting more studies to address the problems.

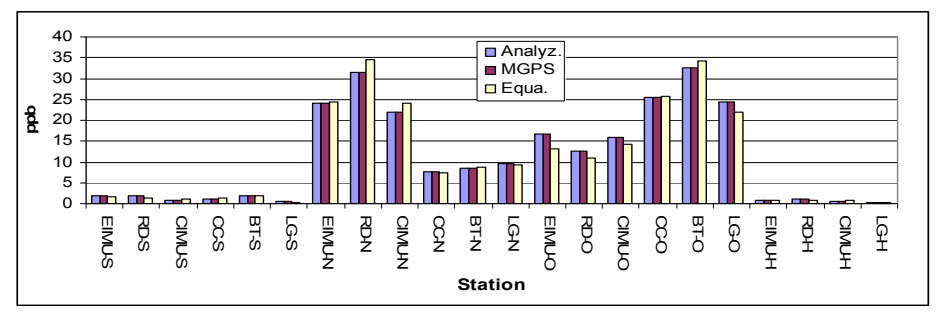

Figure 6: Concentration comparisons of four air pollutants obtained by analyzer, MGPS and equation. 


\section{Applications}

The MGPS has been used in many projects across Canada and in the world indoor and outdoor. One project - Southeast Saskatchewan Airshed Association (SESAA) is located in southern Saskatchewan.

There are many human being activities in the $38,000 \mathrm{~km}$ area including oil and gas industries, power generation, agriculture, transportation etc. Air quality is a big concern by local communities. 30 AMEC passive sampler stations were installed in the area (Figure 7) monitoring $\mathrm{SO}_{2}, \mathrm{NO}_{2}, \mathrm{O}_{3}$, and $\mathrm{H}_{2} \mathrm{~S}$.

The AMEC multigas passive samplers have been used in the airshed for almost 4 years. Very reasonable results have been obtained.

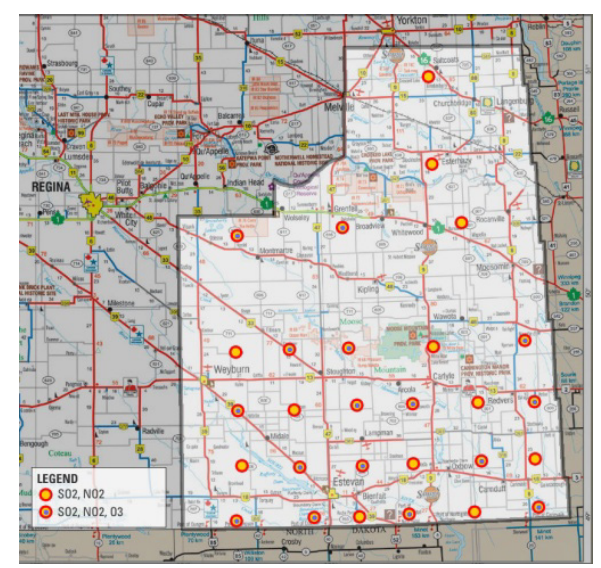

Figure 7: AMEC passive stations in the SESAA.

This project also demonstrates the cost effective feature of MGPS (Table 5). Compared to NPS, MGPS saves about $70 \%$ of major costs.

\section{Conclusions}

A new generation of passive sampling technology - multigas passive sampling system (MGPS) has been developed. Field studies and applications have proved that the MGPS is environment friendly, more cost effective, more convenient to use, and more accurate. The MGPS is a new useful tool in the air monitoring sector for indoor and ambient atmosphere.

\section{References}

[1] Fox, C.B.; Ozone and Antozone, J. and A. Churchill, London. 1873.

[2] Palmes, E.D.; Gunison, A.F.; Personal monitoring device for gaseous contaminants, Am. Ind. Hyg. Assoc. 34, pp. 78-81, 1973. 
[3] Leaderer, B.P.; Koutrakis, P.; Wolfson, J.M.; Sullivan, J.R.; Development and evaluation of a passive sampler to collect nitrous acid and sulphur dioxide, J. Expos. Environ. Epidemiol. 4, 503-511.

[4] Gair, A.J.; Penkett, S.A.; Ovola, P.; Development of a Simple Passive Technology for the Determination of Nitrogen Dioxide in Remote Continental Locations, Atmos. Environ. 25, pp. 1927-1939, 1994.

[5] Mulik, J.D.; Williams, D.; Development of a Simple Passive Technology for the Determination of Nitrogen Dioxide in Remote Continental Locations, Proceedings of the 1986 EPA/APCA symposium of measurement of toxic air pollutants, Raleigh, NC pp. 61-79, 1986.

[6] Kring, E.V.; Damrell, D.J.; Henry, T.J.; Demoor, H.M.; Basilio, A.N.; Simon, C.E.; Laboratory validation and field verification of a new passive colorimetric air monitoring badge for sampling hydrogen-sulfide in air, Am. Ind. Hyg. Assoc. J. 45, pp. 1-9, 1984.

[7] McKee, E.S.; Mcconnaaughey, P.W.; Laboratory validation of a passive length-of-stain dosimeter for hydrogen-sulfide, Am. Ind. Hyg. Assoc. J., 47, pp. 475-481, 1986.

[8] Ogawa \& Company USA Inc. No-NO2 simultaneous sampling protocol, June 1994.

[9] Grosjean, D.; Mohamed, W.M.; A Passive Sampler for Atmospheric Ozone, $J A \& W M A, 42$, pp. 169-173, 1992.

[10] Bamberger, R.L.; Esposito, G.G.; Jacobs, B.W.; Podolak, G.E.; Mazur, J.F.; New passive sampler for organic vapour, Am. Ind. Hyg. Assoc. J. 39, pp. 701-708, 1978.

[11] Brown, V.M.; Crunp, D.R.; Gadiner, D.; Gavin, M.; Assessment of a passive sampler for the determination of aldehydes and ketones in indoor air, Environ. Technol. 15, pp. 679-685, 1994.

[12] Tang, H.; Brassard, B.; Brassard R.; Peake, E.; A new passive sampling system for monitoring SO2 in the atmosphere, FACT, 1, pp. 307-315, 1997.

[13] Tang, H.; Lau, T.; Brassard B.; Cool, W., A new all-season passive sampling system for monitoring $\mathrm{NO}_{2}$ in air, FACT, 6, 338-345.

[14] Tang, H. and Lau, L., A new all season passive sampling system for monitoring ozone in air, Environ. Monit. Assess. 65, 129-137. 1999.

[15] Tang, H.; Sandeluk, J,; Lin L,; and Lown W.; A new all-season passive sampling system for sampling $\mathrm{H}_{2} \mathrm{~S}$ in air, The Scientific World, 2, pp. 155 168. 2002.

[16] Krupa, S.; Legge, A.; Passive sampling of ambient, gaseous air pollutants: an assessment from an ecological perspective, Environmental Pollution, 07, pp. 31-45. 2000,

[17] Higuchi, K.; Schaeffer, D.R.; Hirano, K.; Advanced monitoring method for air environment by Ogawa Passive, in Proceedings of Passive sampling workshop and symposium, Reston, VA, April 2007.

[18] Reid, P.; The role of passive ambient monitoring for sulphur dioxide in the northern Rocky Mountain foothills, in the proceedings of CPANS conference, Edmonton, AB, Canada, 2001. 